\title{
Erratum to: Fluorescence spectroscopy as a potential metabonomic tool for early detection of colorectal cancer
}

\author{
Anders Juul Lawaetz • Rasmus Bro • \\ Maja Kamstrup-Nielsen • Ib Jarle Christensen • \\ Lars N. Jørgensen • Hans J. Nielsen
}

Published online: 4 June 2011

(C) Springer Science+Business Media, LLC 2011

\section{Erratum to: Metabolomics}

DOI 10.1007/s11306-011-0310-7

The original version of this article unfortunately contained a mistake. In the Materials and methods section the number of samples ready for data analysis is incorrectly given as 301, 295 and 300 for the three groups. The correct numbers are 299, 299 and 289. The incorrect numbers come from an intermediate step in the analysis where some irrelevant standard samples were included. Similar quality of results was obtained on datasets of the size incorrectly given in the paper. The data from the paper can be downloaded from our homepage. http://www.models.life.ku.dk/datasets.

The online version of the original article can be found under doi:10.1007/s11306-011-0310-7.

\author{
A. J. Lawaetz $(\bowtie) \cdot$ R. Bro · M. Kamstrup-Nielsen \\ Department of Food Science, Quality and Technology, Faculty \\ of Life Sciences, University of Copenhagen, Rolighedsvej 30, \\ 1958 Frederiksberg C, Denmark \\ e-mail: ajla@life.ku.dk
}

\section{J. Christensen}

Finsen Laboratory, Copenhagen Biocenter, Copenhagen,

Denmark

\section{N. Jørgensen}

Department of Surgery, Bispebjerg Hospital, University

of Copenhagen, Copenhagen, Denmark

\section{H. J. Nielsen}

Department of Surgical Gastroenterology, Copenhagen

University Hospital Hvidovre, Hvidovre, Denmark 